\title{
Optimum Design of Runner System for Router Cover Based on Mold Flow Analysis Technology
}

\author{
Tangqing Kuang and Wenjuan Gu \\ School of Mechanical and Electrical Engineering, \\ East China Jiaotong University, Nanchang 330013, China \\ kuangtq@yahoo.cn
}

\begin{abstract}
During the design of a two-cavity injection mould for router covers, two plans, single-gate plan and dual-gates plan, were setup based on the results of best gate location analysis combined with other aspects. Then fill analysis for the two plans were carried out. The dual-gates plan was selected as an optimal choice for its smaller clamping force, wall shear stress and injection pressure in comparison with the single-gate plan. Although weld lines and air trap exists between the two gates, but they can be eliminated or just have a little effect on the quality of parts. It indicated that the technology of mold flow analysis can be used to optimize the design of gate location and gates number and assist the engineers to improve the quality of their designs of mould.
\end{abstract}

Keywords: Mold flow analysis, Gate location, optimum design.

\section{Introduction}

The use of plastic has been spread to all walks of life, about $80 \%$ plastic production used injection molding method. Therefore, improving the design quality is the key to improve the products quality, productivity and reduce production cost. For a given part design, the runner system design is one of the key issues of its mold design. The complexity of mold structure and cost of mold are determined by the form of gating system directly [1]. Section size and length of the runner system affect the pressure loss of melt flow directly $[2,3]$. Gates location and its number affect the flow behavior of melt in the cavity and eventually affect the quality of product [4]. Using traditional methods which rely on the experience to design gating system can not gain a better runner system, and if we encounter a complex part or not covered before, the gate design can not success in once, we often have to go through a trial mode-modify-try mode-and the modify the phase, that is a great waste for time and cost. With the CAE technology for injection molding developing, mold flow analysis has been successfully used in injection mold design process [5]. By means of mold flow analysis, the optimized gating system becomes a reality. By the flow analysis for different proposals of gating system and comparison of the analysis results such as filling status, weld line location, air trap location, injection pressure, clamping force and other results, the optimal proposal achieved. That can aid the engineers to optimize their design.

Take a router cover for an example, the procedure of optimization runner system of injection mold by mold flow analysis techniques was illuminated in this paper. 


\section{Modeling and Meshing}

The router cove is $430 \mathrm{~mm}$ long, $81 \mathrm{~mm}$ wide and $28 \mathrm{~mm}$ height and shown in Figure 1. And the model was output in iges surface format for the convenient of meshing in Moldflow Plastic Insight (see Ref. 1), software of mold flow analysis for injection molding.

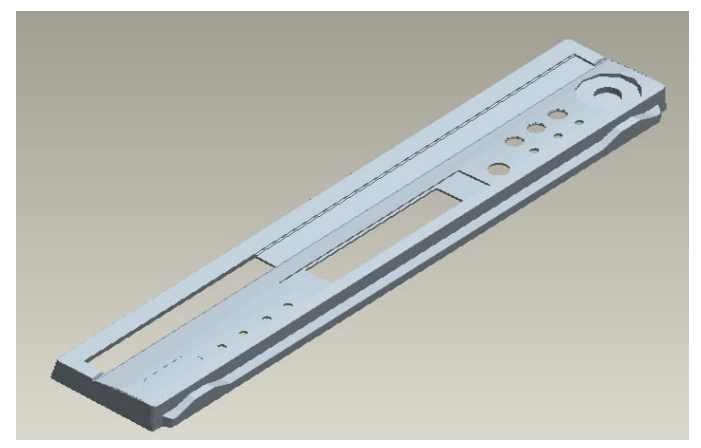

Fig. 1. Router cover model

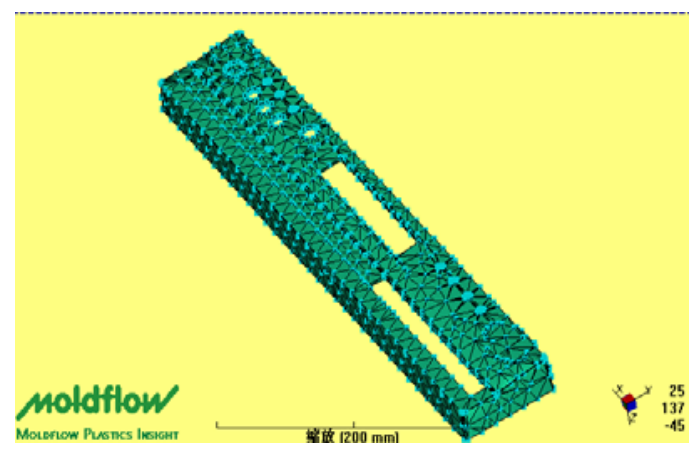

Fig. 2. Meshed router cover model

The iges model was imported into MPI and meshed in fusion type with 3785 elements and 1869 nodes, which shown in figure 2.

\section{Gate Location Analysis and Programming}

Gate location analysis was conducted for the model and the result shown in Figure 3. The blue area shows the suggested best gate location for the cover. The gate location analysis does not take into account some practical considerations for the gate location such as the type of tool, or limitations on the gate location due to part aesthetics etc. The router cover is limited to a 2-plate tool with a cold runner system. As a result of that tooling restriction, the suggested best gate location for the cover, in the middle of the part, is not possible. These results can only be used as a guide to place a gate on the parting line. 


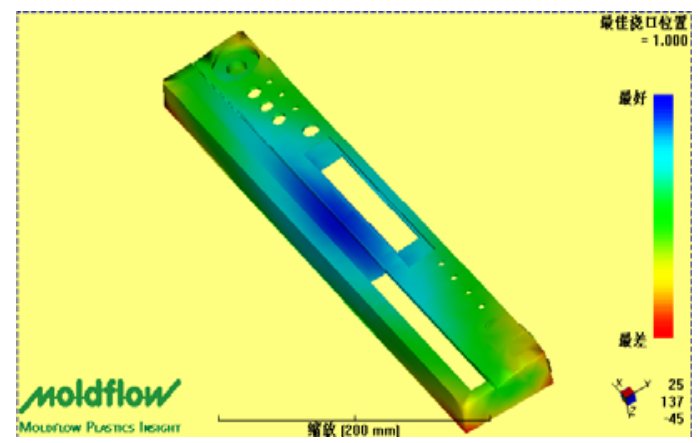

Fig. 3. Best gate location result

For a long kind of part, two-cavity mold layout is a rather good choice. Considering parting location of the router cover, cavity layout of the mold, appearance requirements of the product, simplification and reliability of the mold structure and the best gate location result, single side gate was adopted and it locates at the middle of the part in length direction, close to the best gate location result. Considering the larger size of the part in length direction, another plan which dual side gates locate at the sides of the best gate location result is proposed. Single-gate and dual-gates runner system are shown in Figure 4 and 5 respectively.

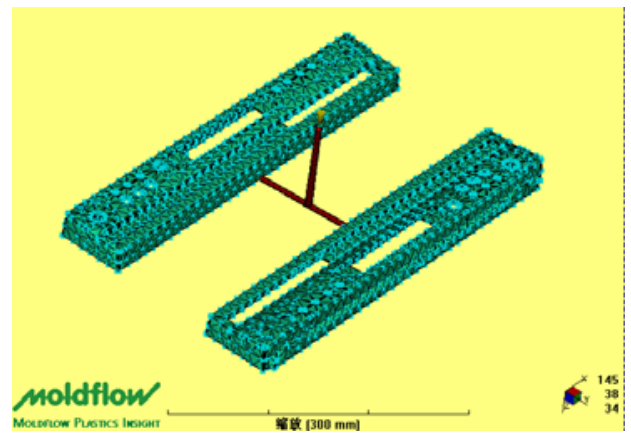

Fig. 4. Single-gate plan

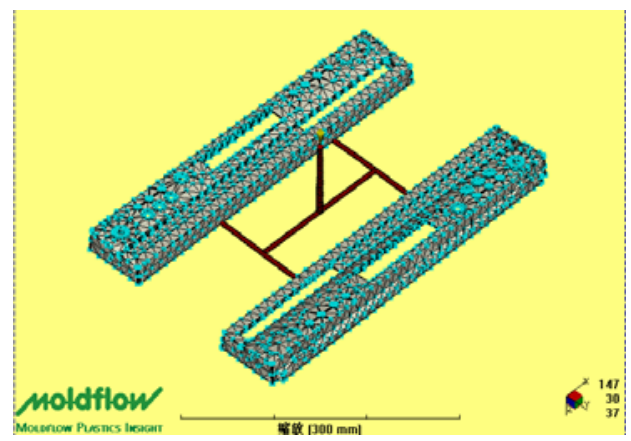

Fig. 5. Dual-gates plan 


\section{Comparison and Analysis of Plans}

\subsection{Process Parameters Setting}

ABS material was used to meet the requirement of strength and hardness of the part. Lanxess ABS 1146 of Lanxess Corporation is selected here. For comparison, the two programs are used same process parameters, mold temperature $80{ }^{\circ} \mathrm{C}$, material

圖 昭
Fill time

$=1.453[\mathrm{~s}]$

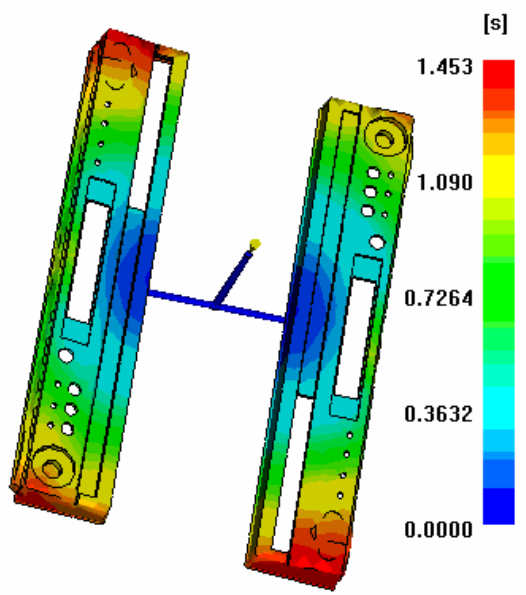

(a)

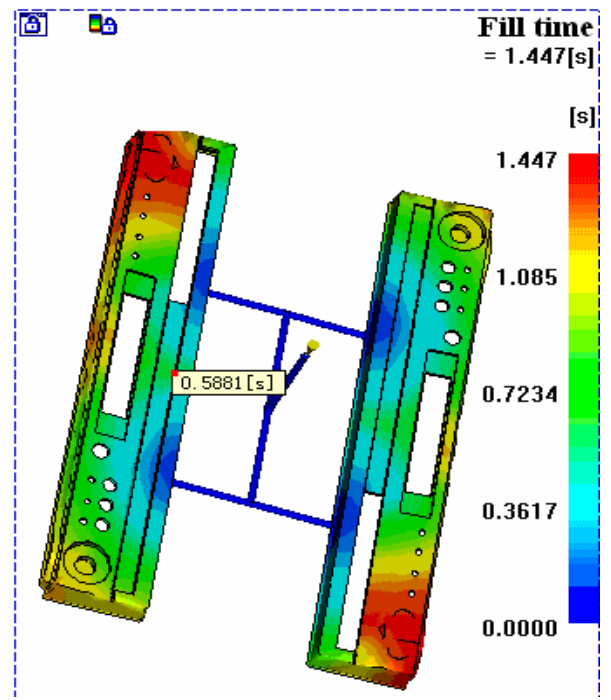

(b)

Fig. 6. Fill time of single-gate plan (a) and dual-gates plan (b) 
temperature $260^{\circ} \mathrm{C}$, flow rate $200 \mathrm{~cm} 3 / \mathrm{s}$; speed / pressure switch is set to $100 \%$ of the filling volume. The better program is chosen by comparing the analysis results of the two programs such as filling time, the pressure distribution at the end of filling time, the distributions of weld line and air trap, the maximum injection pressure and clamping force, the maximum wall shear stress and so on.

\subsection{Comparison of the Analysis Results of the Two Programs}

The fill time result shows the progression of the flow front. Figure 6 (a) and (b) show the fill time results of single-gate plan and dual-gates plan respectively. They indicate that the filling time of the two plans are all about $1.45 \mathrm{~s}$ and they all filling completely. The flow fronts meet at $0.59 \mathrm{~s}$ in dual gates plan.

Pressure at the end of fill will affect the residual stress in the molding parts, the greater the pressure, the greater the residual stress. If the pressure is uneven, the larger the difference, the more uneven the residual stress, the larger the warpage consequently. So the pressure at the end of fill needs to be uniform as far as possible. Pressure distribution at the end of filling time of single-gate plan and dual-gates plan are shown in figure 7 (a) and (b) respectively. The pressure difference is about $85 \mathrm{MPa}$ in single-gate plan while it's 63MPa in dual-gates plan. Pressure distribution in dualgates plan is more uniform than that in single-gate plan.

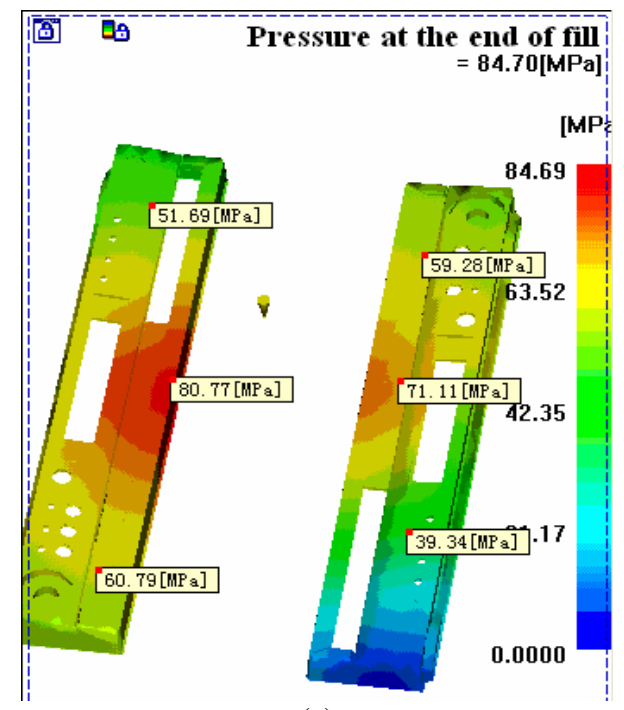

(a)

Fig. 7. Pressure distribution at the end of filling time of single-gate plan (a) and dual-gates plan (b) 


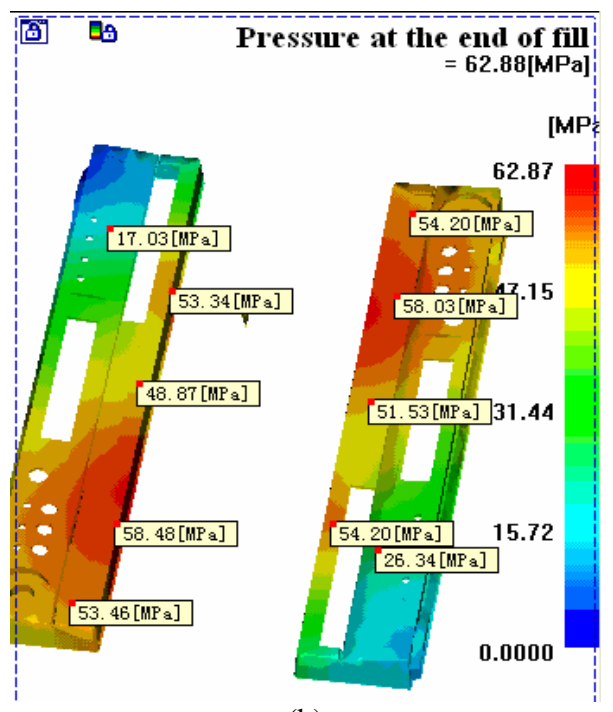

(b)

Fig. 7. (continued)

Weld lines occur where two or more flow fronts converge. The presence of weld lines may indicate a structural weakness and/or a surface blemish. Weld line strength is influenced by the temperature at which the weld line is formed and the pressure exerted on the weld while the material is within its recommended processing temperature range. Weld line results with the flow front temperature highlightened of singlegate plan and dual-gates plan are shown in figure 8 (a) and (b) respectively. In the two plans, weld lines exist near the rectangular hole and small radial holes. But all the flow front temperature at weld lines are about $260^{\circ} \mathrm{C}$ which is melt injected temperature. In comparison with single gate plan, an extra long weld line exists between the two gates in dual-gates plan, but the flow front temperature and the pressure exerted on them are high, so the weld line strength is ensured and it has less negative impact on part quality.

Air trap occurs at the ends of flow paths or where the melt stops at a convergence of at least two flow fronts. The presence of weld lines may indicate a structural weakness and/or a surface blemish and should be avoid by taking some measures. Figure 9 (a) and (b) show the air trap distribution of single-gate plan and dual-gates plan respectively. In comparison with single gate plan, extra air traps exist between the two gates in dual-gates plan. The air trap existing where the melt stops at a convergence of several flow fronts can be eliminated by setting knockout pins or adopting the mosaic of core there. And the air trap existing at the ends of flow paths can be eliminated by the venting at the parting plane. 
Temperature at flow front [C]

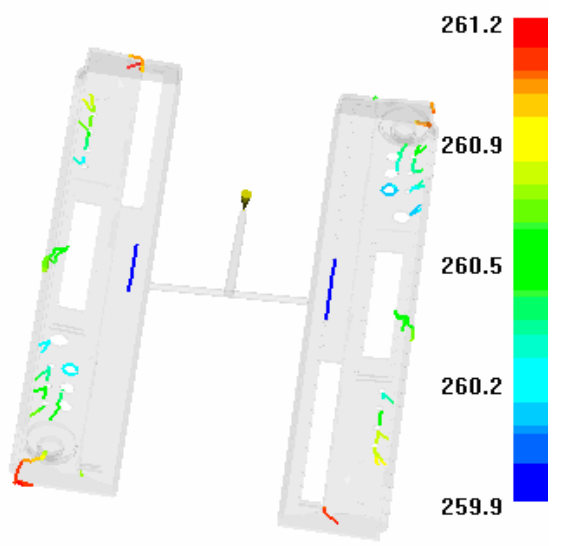

(a)

圆 时 Weld lines

Temperature at flow front $=261.1[\mathrm{C}]$

Temperature at flow front [C]

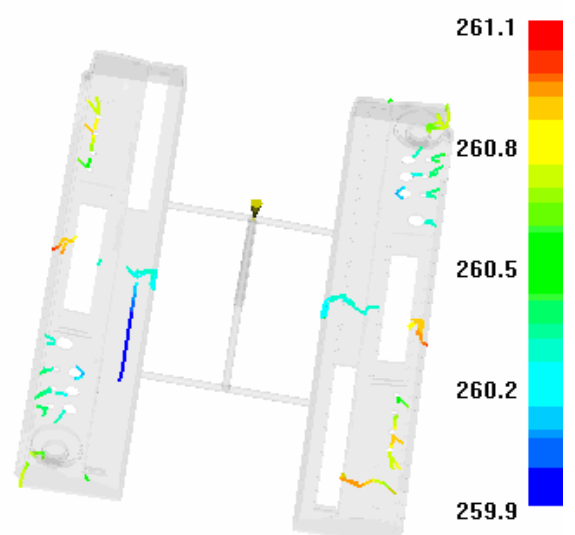

(b)

Fig. 8. Weld line distribution of single-gate plan (a) and dual-gates plan (b) 
圖绝

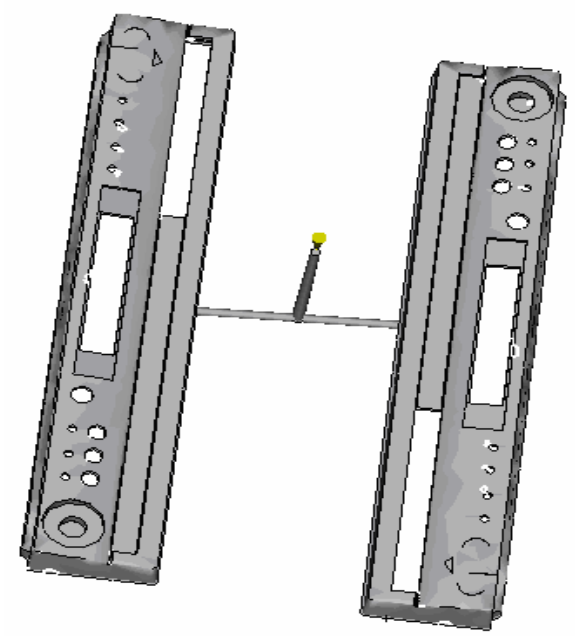

(a)

圖明

Air trap

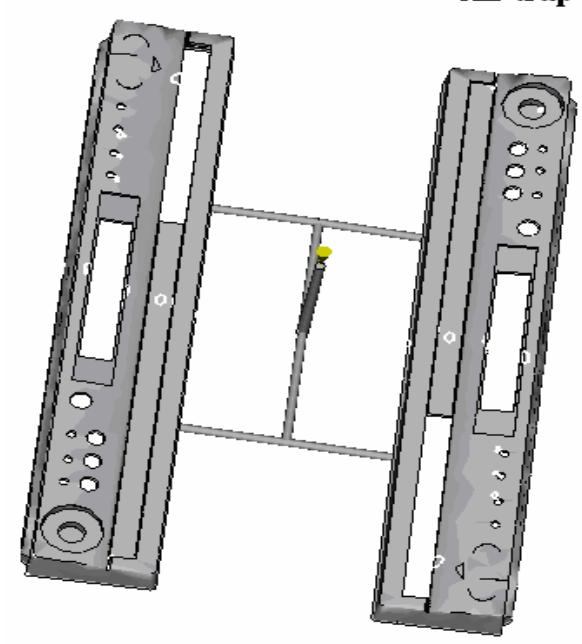

(b)

Fig. 9. Air trap distribution of single-gate plan (a) and dual-gates plan (b)

The selected injection molding machine must meet the requirements of the maximum injection pressure and clamping force. The higher the maximum injection pressure and clamping force are, the more the energy consumption is and the higher the injection molding machine specifications is. Pressure trace at the injection location of single-gate plan and dual-gates plan are shown in figure 10 (a) and (b) respectively. The maximum injection pressure in single-gate plan is $114 \mathrm{MPa}$ while it's $106 \mathrm{MPa}$ in 
dual-gates plan. Figure 11 (a) and (b) show the clamp force development of singlegate plan and dual-gates plan respectively. The maximum clamp fore in single-gate plan is 344 ton while it's 259 ton in dual-gates plan.

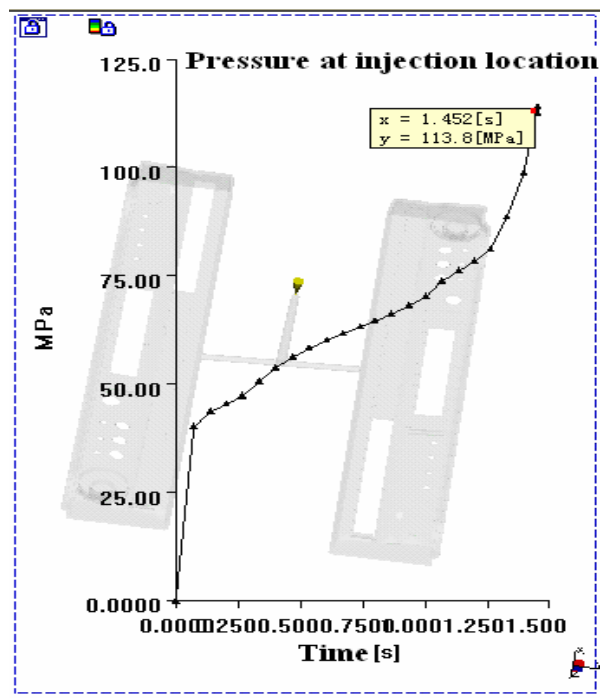

(a)

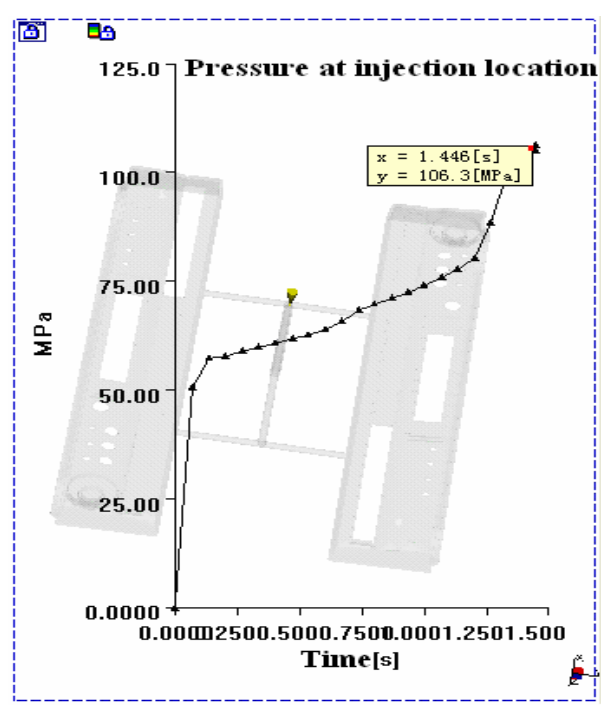

(b)

Fig. 10. Pressure trace at the injection location of single-gate plan (a) and dual-gates plan (b) 


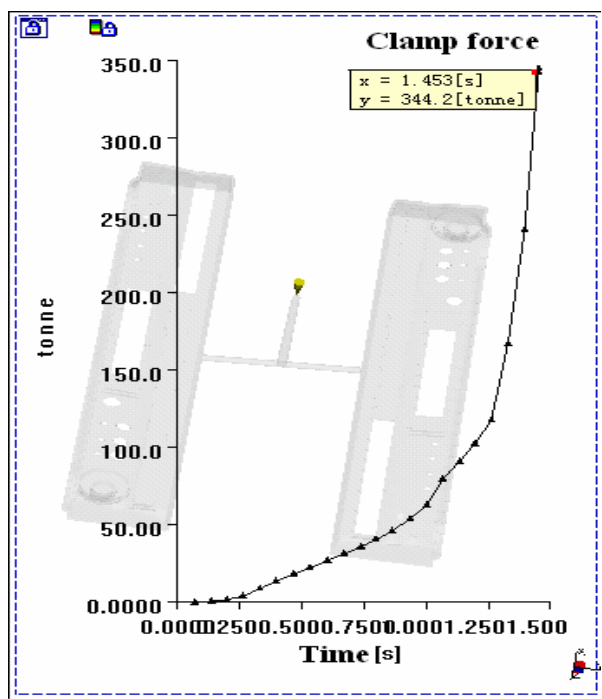

(a)

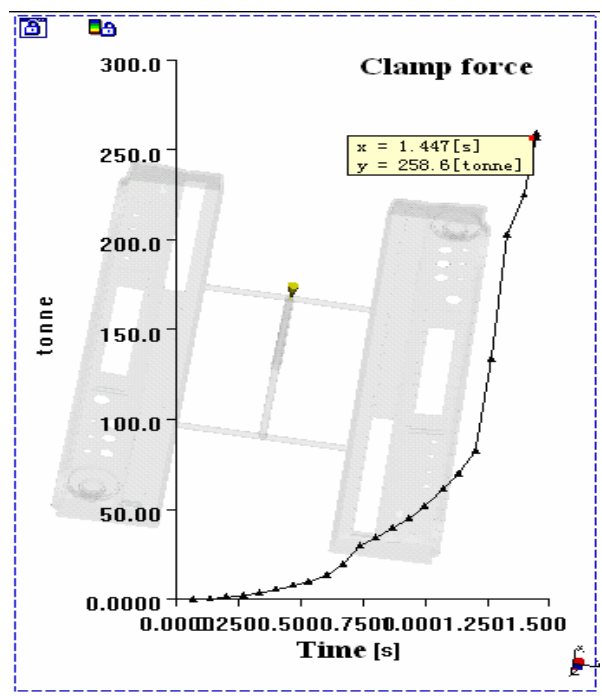

(b)

Fig. 11. Clamp force of single-gate plan (a) and dual-gates plan (b)

Shear flow is the main flow behavior during the filling stage of injection molding and the maximum shear stress exist near the wall. The wall shear stress needs to be below the maximum allowable shear stress of the used material as far as possible to avoid material degradation for the excessive shear stress. The maximum allowable shear stress of selected material is $0.25 \mathrm{MPa}$.

The analysis log result shows that the maximum shear stress and average shear stress in single-gate plan are $0.922 \mathrm{MPa}$ and $0.185 \mathrm{MPa}$ respectively. The maximum 
shear stress and average shear stress in dual-gates plan are $0.816 \mathrm{MPa}$ and $0.173 \mathrm{MPa}$ respectively and they all less than the values of single-gate plan. Shear stress at wall of single-gate plan and dual-gates plan are shown in figure 12 (a) and (b). As we can see, most of the shear stress at wall of dual-gates plan are below the allowable shear stress $0.25 \mathrm{MPa}$ while shear stress at wall at a large area of single-gate plan exceeds the allowable value.

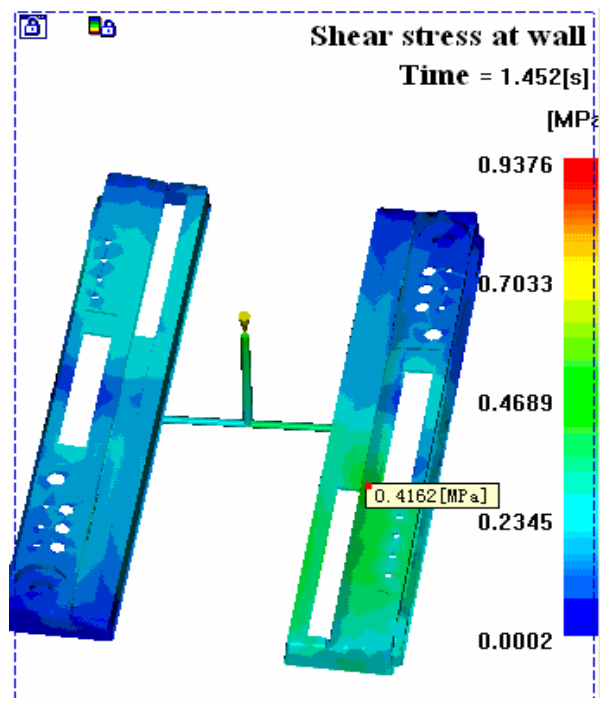

(a)

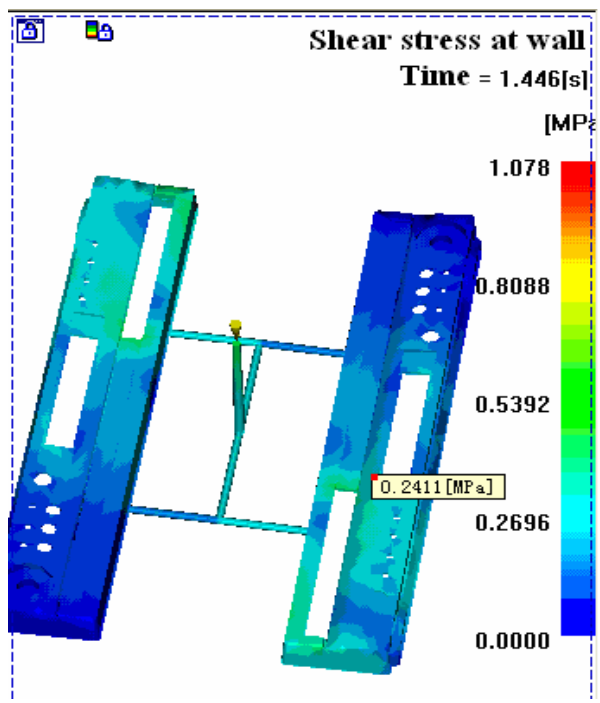

(b)

Fig. 12. Shear stress at wall of single-gate plan (a) and dual-gates plan (b) 


\subsection{Choice}

In comparison with the single-gate plan, the dual-gates plan has smaller clamping force, wall shear stress and injection pressure. Although weld lines and air trap exists between the two gates, but they can be eliminated or just have a little effect on the quality of parts. So the dual-gates plan is selected as an optimal choice.

\section{Conclusion}

In this paper, comparison and selection of different gate settings is conducted by mold flow analysis. Finally we get the best gate location and gate panel number for the router which is the author's major work, The length and cross section size can be determined by the following choice of mold plate and the mold specifically designs. The runner section size can be optimized by runner balance analysis. And the gate section size can be optimized to ensure the full compensation by packing analysis and cooling analysis. The resulting optimum runner system can be used to improve product quality.

From this example, we know that the successful application of mold flow analysis for injection mold design provides the scientific basis and reference for mold designers and aids designers to optimize the design programs, reduce testing time, and improve qualities of mold and products.

\section{Acknowledgments}

This work forms part of a project supported by the Education Department of Jiangxi Province with grant GJJ10126.

\section{References}

1. Beaumont, J.P.: Runner and Gating Design Handbook: Tools for Successful Injection Molding. Hanser Gardner Publications (2004)

2. Lee, K.S., Lin, J.C.: Design of the runner and gating system parameters for a multi-cavity injection mould using FEM and neural network. The International Journal of Advanced Manufacturing Technology, Published by Elsevier Science Ltd, (2006)

3. Zhai, M., Lam, Y.C., Au, C.K.: Runner sizing and weld line positioning for plastics injection moulding with multiple gates. In: Engineering with Computers. Springer Publishing, Heidelberg (2006)

4. Lee, J., Lee, J.: Gate positioning design of injection mould using bi-objective micro genetic algorithm. Proceedings of the Institution of Mechanical Engineers, Part B: Journal of Engineering Manufacture, Professional Engineering Publishing (2008)

5. Moldflow Corporation: Simulation Fundamentals-An Introduction for MPI 6.0. Moldflow Corporation, Boston, America (2006) 